\title{
Increased Release of Proinflammatory Proteins in Primary Human Adipocytes and Activation of the Inflammatory NFkB, p38, and ERK Pathways upon Omentin Treatment
}

\author{
Corinna Niersmann ${ }^{a, b} \quad$ Karin Röhrig ${ }^{a, b}$ Matthias Blüher ${ }^{b, c}$ \\ Michael Roden ${ }^{a, b}, d \quad$ Christian Herder ${ }^{a, b}, d \quad$ Maren Carstensen-Kirberg ${ }^{a, b}$ \\ a Institute for Clinical Diabetology, German Diabetes Center, Leibniz Center for Diabetes \\ Research at Heinrich Heine University Düsseldorf, Düsseldorf, Germany; ${ }^{b}$ German Center \\ for Diabetes Research (DZD), Munich-Neuherberg, Germany; ${ }^{\mathrm{C} D e p a r t m e n t}$ of Medicine, \\ University of Leipzig, Leipzig, Germany; ${ }^{d}$ Division of Endocrinology and Diabetology, \\ Medical Faculty, Heinrich Heine University, Düsseldorf, Germany
}

\section{Keywords}

Omentin · Adipokine $\cdot$ Primary human adipocytes · Proinflammatory action · NFKB signaling

\begin{abstract}
Objectives: To investigate the impact of omentin on the release of inflammation-related biomarkers and inflammatory pathways in primary human adipocytes. Methods: Adipocytes were treated with or without omentin (500 and 2,000 ng/mL), and the supernatants were analyzed for inflammation-related biomarkers using proximity extension assay technology. Potential upstream regulators of the omentin-stimulated proteins were identified using Ingenuity Pathway Analysis. Protein levels of components of inflammatory pathways were measured using Western blotting. Results: $2,000 \mathrm{ng} / \mathrm{mL}$ omentin induced the release of 30 biomarkers $97.1 \pm 31.1$-fold in the supernatants (all $p<0.05$ ). Most biomarkers were proinflammatory chemokines and cytokines. We identified the transcription factor nuclear factor "kappa-light-chain-enhancer" of activated B cells (NFkB) and the kinases p38 and extracellular signal-regulated kinase (ERK)1/2 as potential upstream regulators in silico. On the cellular level, treatment with $2,000 \mathrm{ng} / \mathrm{mL}$ omentin for $24 \mathrm{~h}$ enhanced the phosphorylation levels of NFKB $2.1 \pm 0.3$-fold ( $p<0.05$ ), of p38 $2.6 \pm 0.4$-fold $(p<0.05$ ), and of ERK1/2 $1.8 \pm 0.2$-fold $(p<0.05)$. Conclusions: These data argue that omentin exerts proinflammatory effects through the activation of the inflammatory NFKB, p38, and ERK1/2 pathways in cultured primary adipocytes.


Niersmann et al.: Proinflammatory Action of Omentin in Human Adipocytes

\section{Introduction}

Omentin (also referred to as intelectin-1) is a secreted adipokine which is abundantly expressed in the stromal vascular fraction of visceral fat depots, but hardly detectable in subcutaneous adipose tissue. Furthermore, omentin is produced in other tissues including the heart, lung, or intestine [1].

Several cross-sectional studies found an inverse association between circulating omentin and multiple risk factors for type 2 diabetes (T2D) and cardiovascular diseases (CVD) such as increased waist circumstance, elevated blood pressure, dyslipidemia, or impaired glucose tolerance [2-8]. Omentin levels were also decreased in patients with coronary artery disease or ischemic stroke $[6,9-11]$. In agreement with these studies, omentin exerted protective effects on human umbilical vein endothelial cells (HUVECs) and smooth muscle cells (SMCs) [12-16]. In cultured human adipocytes, omentin induced the phosphorylation of protein kinase B (also known as Akt) and stimulated glucose uptake in response to insulin [1]. The postulated atheroprotective phenotype of omentin from in vitro studies was supported by mouse models showing that omentin improved ischemia-induced revascularization and reduced the formation of aortic atherosclerotic lesions, cardiac hypertrophy, and myocardial ischemic damage [14,17-19].

In contrast, prospective analyses showed positive associations of omentin levels with both higher risk of T2D $[20,21]$ and cardiovascular events in population-based samples and in patients with preexisting CVD [22-24]. Thus, these data point towards the possibility that omentin levels may contribute to cardiometabolic risk in humans.

We recently performed a liquid-chromatography mass spectrometry-based proteomics study to investigate the effect of omentin on the secretome of human adipocytes. The analysis of omentin-regulated proteins in metabolic and cellular signaling pathways revealed that these proteins were enriched in pathways indicating a proinflammatory state, cellular stress, and a crosstalk with other tissues [25]. However, this study did not provide detailed knowledge on the mechanisms by which the omentin-induced secretory process is mediated.

Therefore, the overall aim of the present study was to extend the findings of the effects of omentin on primary human adipocytes and to identify the signaling pathways activated by omentin using Western blotting. Especially, we aimed to validate the in silico data from our proteomics study indicating an involvement of nuclear factor "kappa-light-chain-enhancer" of activated B cells (NFKB) in the mode of action of omentin. In detail, the aims of this study were (1) to examine the effect of omentin on the secretion and expression of biomarkers of inflammation in primary adipocytes, (2) to identify potential upstream regulators of the omentin-induced secretion profile, and (3) to characterize the signaling cascades which mediate the effects of omentin.

\section{Materials and Methods}

\section{Cell Culture and Treatment of Primary Human Adipocytes}

Primary human preadipocytes isolated from subcutaneous adipose tissue from 5 nondiabetic donors were purchased from Lonza (Basel, Switzerland) and PromoCell (Heidelberg, Germany). Approval by an ethics committee was not required. Cells from each donor (no cell pools) were used at passage three and incubated under sterile conditions at $37^{\circ} \mathrm{C}$ and $5 \% \mathrm{CO}_{2}$. The cells were cultivated as described [25]. The differentiation process from fibroblast-like preadipocytes into lipid-loaded adipocytes was monitored using light microscopy, and the AdipoRedTM Assay Reagent from Lonza was applied to stain intracellular lipids. After differentiation of preadipocytes into mature adipocytes, cells were starved in adipocyte nutrition 
medium (PromoCell) containing $50 \mu \mathrm{g} / \mathrm{mL}$ gentamicin and $8 \mu \mathrm{g} / \mathrm{mL}$ D-biotin. After $4 \mathrm{~h}$, the medium was replaced by the same medium, and adipocytes were treated with 500 or 2,000 $\mathrm{ng} / \mathrm{mL}$ omentin (CellSystems, Troisdorf, Germany) for $30 \mathrm{~min}$ and $24 \mathrm{~h}$. We also included stimulation with $20 \mathrm{ng} / \mathrm{mL}$ tumor necrosis factor- $\alpha$ (TNF- $\alpha$ ) (Cell Signaling Technology, Danvers, MA, USA) for $24 \mathrm{~h}$ as positive control. The concentration of $500 \mathrm{ng} / \mathrm{mL}$ omentin is close to the median level in a nondiabetic population, and the dose of $2,000 \mathrm{ng} / \mathrm{mL}$ omentin represents the upper physiological range of what can be observed in the general population [4, 20]. Endotoxin levels were below $0.5 \mathrm{EU} / \mathrm{mL}$ (corresponding to below $0.1 \mathrm{ng} / \mathrm{mL}$ ) at an omentin concentration of 2,000 ng/mL as assessed using the QCL-1000TM Endpoint Chromogenic LAL assay (\#50-647U; Lonza). Recombinant human omentin and TNF- $\alpha$ were first dissolved in sterile, distilled water (Roth, Karlsruhe, Germany) and diluted in phosphate-buffered saline (Thermo Fisher Scientific, Waltham, MA, USA) containing $0.1 \%$ bovine serum albumin (BSA; catalogue number A9418, Sigma-Aldrich, Munich, Germany) as carrier protein. This led to BSA concentrations of $0.001 \%$ for the treatment with omentin at $500 \mathrm{ng} / \mathrm{mL}$ and for the treatment with TNF- $\alpha$ and of $0.004 \%$ for the treatment with omentin at 2,000 ng/mL. Differentiated adipocytes without omentin treatment (incubated with $0.001 \%$ BSA) served as negative control. After the incubation time, adipocyte supernatants were collected, centrifuged for $10 \mathrm{~min}$ at $1,800 \times g$ at $4{ }^{\circ} \mathrm{C}$ and transferred to new tubes. The supernatants and the seeded adipocytes in cell culture plates were stored at $-80^{\circ} \mathrm{C}$ until the protein analysis in supernatants or protein extraction from cells was performed. In addition, a nonradioactive colorimetric cell viability assay (WST-8, PromoCell) was applied according to the manufacturer's instructions to estimate the viability of primary human adipocytes after treatment with omentin or TNF- $\alpha$.

\section{Measurement of 92 Inflammation-Related Biomarkers}

Proximity extension assay (PEA) technology (Olink Proteomics, Uppsala, Sweden) was used for high-throughput, multiplex measurement of 92 proteins in the adipocyte supernatants. This technology has been used before to investigate associations between multiple protein biomarkers, T2D, and cardiovascular risk factors [26-28]. The selected biomarker panel (Olink Inflammation I) targets proteins that are involved in biological processes such as chemotaxis, inflammatory response, cell adhesion, regulation of immune response, or extracellular matrix organization [26]. A full list of all 92 biomarkers is given in online supplementary Table 1 (for all online suppl. material, see www.karger.com/doi/10.1159/000506405). Briefly, this protein quantification method combines an antibody-based immunoassay with a polymerase chain reaction (PCR) and quantitative real-time PCR (qPCR) [29]. Data are presented as normalized protein expression units that represent an arbitrary unit on a $\log 2$ scale. The relative quantification units are calculated from the threshold cycle values from the qPCR [29].

\section{Measurement of Proteins Involved in Inflammatory Pathways Using Western Blotting}

Proteins were extracted from primary human adipocytes using RIPA buffer containing $50 \mathrm{mmol} / \mathrm{L}$ Tris-HCl (pH 8.0) (Roth), $150 \mathrm{mmol} / \mathrm{L} \mathrm{NaCl}$ (Roth), 1\% NP-40 (Abcam, Cambridge, UK), $0.5 \%$ sodium deoxycholate (Roth), $0.1 \%$ sodium dodecyl sulfate (Merck, Darmstadt, Germany), 10\% PhosSTOP Phosphatase Inhibitor Cocktail (Roche, Basel, Switzerland), and $10 \%$ cOmplete Mini Protease Inhibitor Cocktail (Roche). After sonication for $10 \mathrm{~s}$ with $60 \%$ pulse, lysates were centrifuged for $60 \mathrm{~min}$ at $17,640 \times \mathrm{g}$ at $4{ }^{\circ} \mathrm{C}$. Protein content of the lysates was quantified using a bicinchoninic acid protein assay (ThermoFisher Scientific) according to the manufacturer's instructions. We determined protein abundance by Simple Western size-based assays using a 12-230 kDa Separation Module (ProteinSimple, San Jose, CA, USA) and analyzed the data as described [30]. The antibody source for phospho-extracellular signal-regulated kinase (ERK) 1 (Thr202/Tyr204)/ERK2 (Thr185/Tyr187) (\#AF1018) was R\&D Systems (Minneapolis, MN, USA). The antibodies for phospho-NFkB p65 (Ser536) 
Niersmann et al.: Proinflammatory Action of Omentin in Human Adipocytes

(81E11) (\#3033), phospho-p38 mitogen activated protein kinase (MAPK) (\#4511) (Thr180/ Tyr182) (D3F9), and the appropriate total antibodies NFkB p65 (C22B4) (\#4764), p38 MAPK (\#9212), and p44/42 MAPK (ERK1/2) (137F5) (\#4377) were purchased from Cell Signaling Technology. Relative phosphorylation levels of NFkB, p38 MAPK, and p44/42 (ERK1/2) were normalized to the levels of the appropriate total proteins.

\section{Statistical and Pathway Analysis}

Prism 7 (GraphPad Software, La Jolla, CA, USA) was used for statistical analyses. All experiments were performed with five biological replicates (i.e., cells from five different donors) if not specified otherwise. Protein levels were represented as mean values with standard error of the mean (SEM). Alterations of protein levels between treatments were analyzed by Friedman's test or ANOVA followed by Dunn's test or the Benjamini-Hochberg correction for multiple testing. $p$ values below 0.05 were considered as statistically significant.

In order to better understand the secretory mechanism potential, upstream regulators of the omentin-regulated proteins were analyzed using Ingenuity Pathway Analysis (IPA) (Qiagen, Hilden, Germany) as described [26]. For the identification of potential upstream regulators, we used fold changes in expression and $p$ values of omentin-regulated proteins that referred to the treatment with $2,000 \mathrm{ng} / \mathrm{mL}$ omentin for $24 \mathrm{~h}$. The analysis gives an activation z-score that predicts the activation state of the potential upstream regulators. A z-score above 2 indicates the activation and a z-score below -2 points towards the inhibition of potential upstream regulators. In addition, Fisher's exact test is designed to reduce the chance that random data will generate significant predictions.

\section{Results}

Omentin Induced the Release of Proinflammatory Protein Biomarkers into Human Adipocyte Supernatants

In supernatants of omentin-treated, TNF- $\alpha$-treated, and untreated primary human adipocytes, 41 out of 92 biomarkers from the multimarker panel were detectable (Fig. 1). Omentin increased the secretion of 17 biomarkers $32.3 \pm 6$. 8 -fold (mean \pm SEM) at $500 \mathrm{ng} / \mathrm{mL}$ omentin (all $p<0.05$ ) and of 30 biomarkers $97.1 \pm 31$.1-fold at 2,000 ng/mL omentin (all $p<0.05$ ). The administration of $20 \mathrm{ng} / \mathrm{mL}$ TNF- $\alpha$ as positive control led to an upregulation of 29 proteins $201.9 \pm 138.7$-fold (all $p<0.05$ ). TNF ligand superfamily member 12 (TWEAK) was downregulated by $500 \mathrm{ng} / \mathrm{mL}$ omentin. The remaining six proteins were not significantly regulated by any of the treatments (Fig. 1).

Of note, the treatment of the human adipocytes with 500 or $2,000 \mathrm{ng} / \mathrm{mL}$ omentin or 20 $\mathrm{ng} / \mathrm{mL}$ TNF- $\alpha$ for $24 \mathrm{~h}$ had no detrimental impact on cell viability (data not shown).

The 30 upregulated proteins by 2,000 ng/mL omentin were classified into four groups (Table 1), and the largest group of co-regulated proteins consisted of 15 chemokines which were upregulated $157.3 \pm 56.4$-fold (all $p<0.05$ ). Six chemokines were upregulated more than 200-fold (209- to 729-fold, all $p<0.0001$ ): monocyte chemotactic protein-3 (MCP-3), C-X-C motif chemokine 6 (CXCL6), CXCL5, CXCL10, MCP-2, and CC-chemokine ligand 20 (CCL20). The remaining nine chemokines were upregulated less than 39-fold ( $p$ between $<0.0001$ and 0.0116 ). As the second largest group, six cytokines were upregulated $39.4 \pm$ 27.2 -fold (all $p<0.05$ ) at $2,000 \mathrm{ng} / \mathrm{mL}$ omentin. The most prominently increased cytokines in this dataset were interleukin-6 (IL-6) and leukemia inhibitory factor (also a member of the IL-6 family) which were upregulated 167- and 61-fold, respectively (both $p<0.0001$ ). 2,000 $\mathrm{ng} / \mathrm{mL}$ omentin also enhanced the release of three proteases $99.9 \pm 64.3$-fold (all $p<0.05$ ). The most strongly increased proteins in this group were matrix metalloproteinase-1 (MMP-1) 


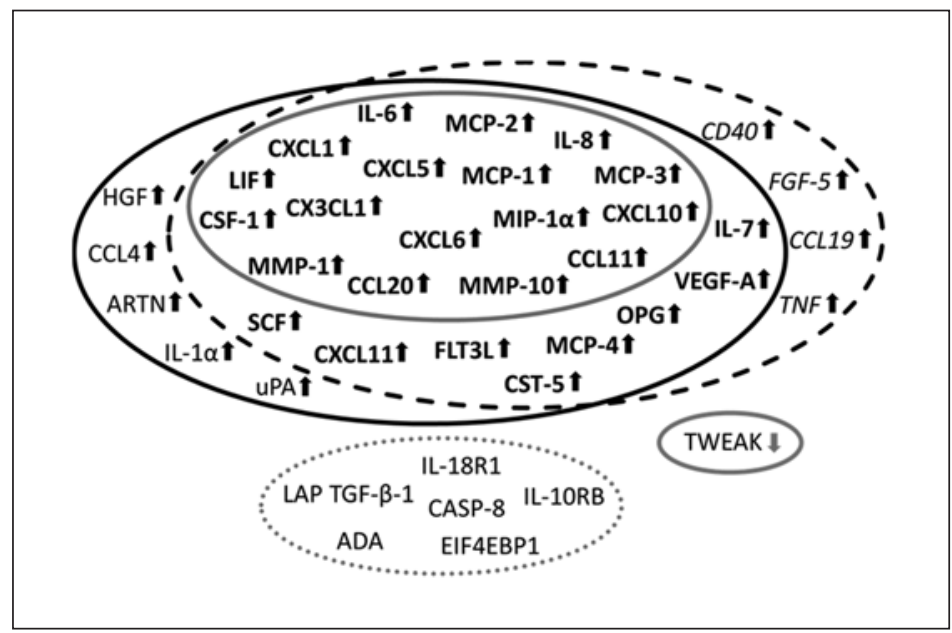

Fig. 1. Overview of inflammation-related proteins that were up-, down-, or not regulated by different concentrations of omentin or TNF- $\alpha$. The grey circle shows inflammation-related mediators regulated by $500 \mathrm{ng} / \mathrm{mL}$ omentin, the black circle by $2,000 \mathrm{ng} / \mathrm{mL}$ omentin, and the dashed black circle by $20 \mathrm{ng} / \mathrm{mL}$ TNF- $\alpha$ as positive control. The overlap between the grey, black, and dashed black circles presents the regulated proteins by three treatments $(p<0.05$ compared to control). Bold print indicates proteins upregulated by both omentin and TNF- $\alpha$, and italic print represents additional biomarkers upregulated by TNF- $\alpha$ only. The biomarkers in the grey dotted circle were not statistically significant upregulated ( $p>0.05$ compared to control). Black and grey arrows show the upregulation or downregulation of the biomarkers. ADA, adenosine deaminase; ARTN, artemin; CASP, caspase; CCL, C-C motif chemokine ligand; CD40, tumor necrosis factor receptor superfamily member 5; CSF-1, macrophage colony-stimulating factor 1; CST-5, cystatin-D; CXCL, C-X-C motif chemokine; CX3CL1, fractalkine; EIF4EBP1, eukaryotic translation initiation factor 4E binding protein 1; FGF, fibroblast growth factor; FLT3L, Fms-related tyrosine kinase 3 ligand; HGF, hepatocyte growth factor; IL, interleukin; IL-10RB, interleukin 10 receptor subunit beta; IL-18R1, interleukin 18 receptor 1 ; LAP TGF- $\beta$-1, latency-associated peptide transforming growth factor beta 1; LIF, leukemia inhibitory factor; MCP, monocyte chemotactic protein; MIP-1 $\alpha$, macrophage inflammatory protein-1 alpha; MMP, matrix metalloproteinase; OPG, osteoprotegerin; SCF, stem cell factor; TNF, tumor necrosis factor; TWEAK, tumor necrosis factor ligand superfamily member 12; uPA, urokinase-type plasminogen activator; VEGF-A, vascular endothelial growth factor A.

and MMP-10, which were upregulated 222- and 74-fold, respectively (both $p$ values $<0.0001$ ). The expression of six other proteins was enhanced $3.1 \pm 0.5$-fold (all $p<0.05$ ) at 2,000 ng/ $\mathrm{mL}$ omentin. The remaining proteins listed in Table 1 were upregulated by TNF- $\alpha$ only or (for six proteins) regulated neither by omentin nor by TNF- $\alpha$.

\section{Omentin Induced a Proinflammatory Intracellular Response}

The IPA analysis software revealed 59 potential upstream regulators of the omentininduced secretion profile. Out of these 59 potential regulators, 56 were predicted to be activated, and the remaining three (HMOX1, Nr1h, and IL1RN) to be inhibited (online suppl. Table 2 for the full list of potential upstream regulators and Table 2 for explanation of abbreviations). Among the top 15 potential upstream activators were five proinflammatory cytokines (TNF, IL1B, IL1A, IL17A, and IL18). The second biggest group consisted of three regulators (ERK1/2, p38 MAPK, and c-Jun N-terminal kinase [JNK]) that were characterized as "groups" by the IPA software. In addition, RNASE1 and RNASE2 belonged to the group of enzymes, and NFKB and immunoglobulin were defined as complexes. Other groups included transcription regulators (RELA and JUN) and kinases (ERBB2). The most relevant potential activators were TNF, IL1B, NFKB, and RNASE2 with 18, 16, 14, and 10 differentially omentinregulated target molecules, respectively. 


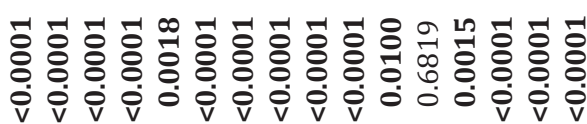

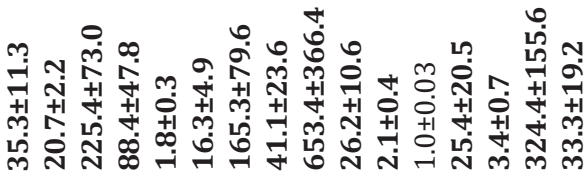

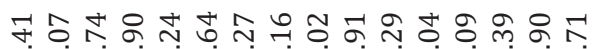
$+\begin{aligned} & i_{1} \\ & +\end{aligned}$

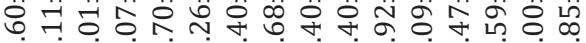
लें

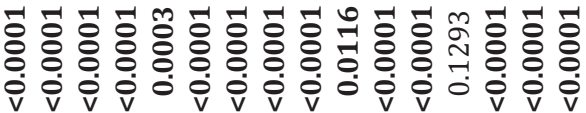

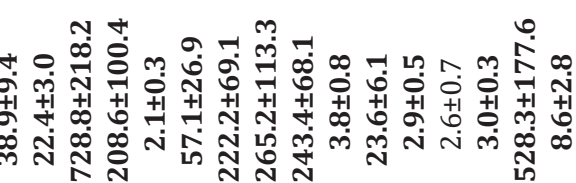
กำ

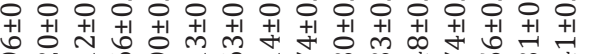

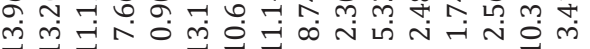

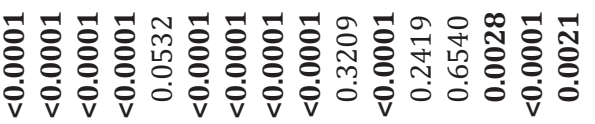

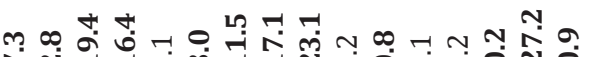

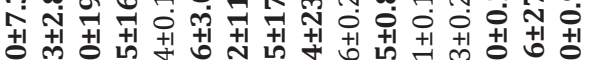

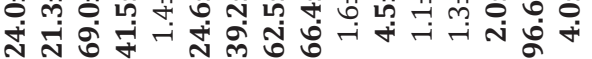

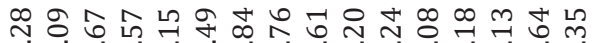

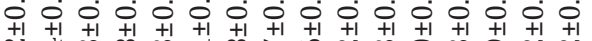

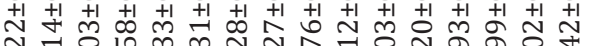
लु

m

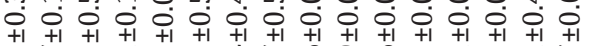

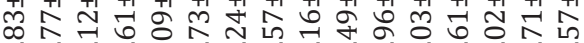

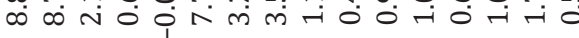

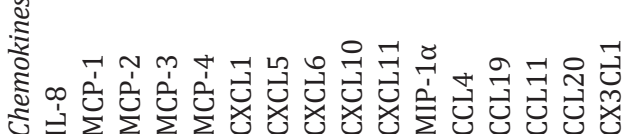

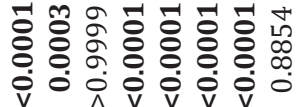

$\begin{array}{ll}1 & 0 \\ 0 & 0 \\ 0 & 0 \\ 0 & 0 \\ 0 & 0 \\ 0 & 0\end{array}$

๓ $N$ *

L

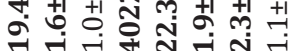

ᄂก

mi

+1 +it o बें

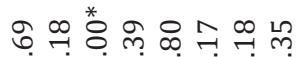

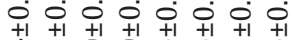

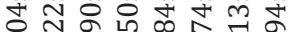

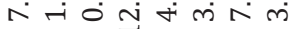

कా

ot $\begin{gathered}+1 \\ +1\end{gathered}$

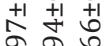
तु के

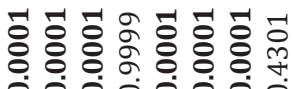

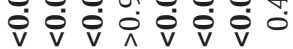

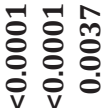

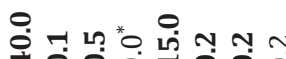

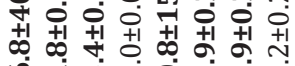
羊

กี นิํำ +1 งิ่

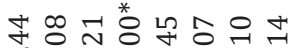

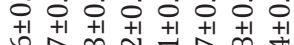

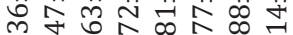
○一

ㅎํ 융ำ

하 㘶

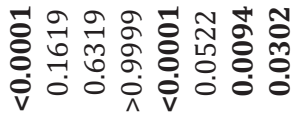

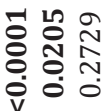

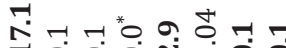

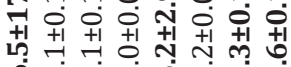
它 $\rightarrow$ -

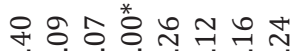

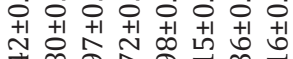

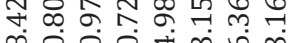

둥

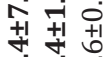
เค่ เค่ ț \& + $\begin{array}{ll}+1 \\ 0\end{array}$

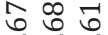
$\exists$ ㅅำ

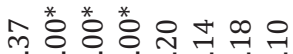

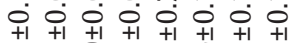
শึ่ ल०००

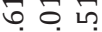
+ $\begin{aligned} & 1+1 \\ & +1\end{aligned}$ ข 0 ก

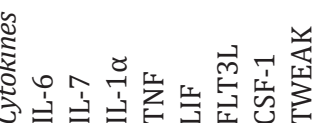

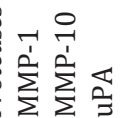




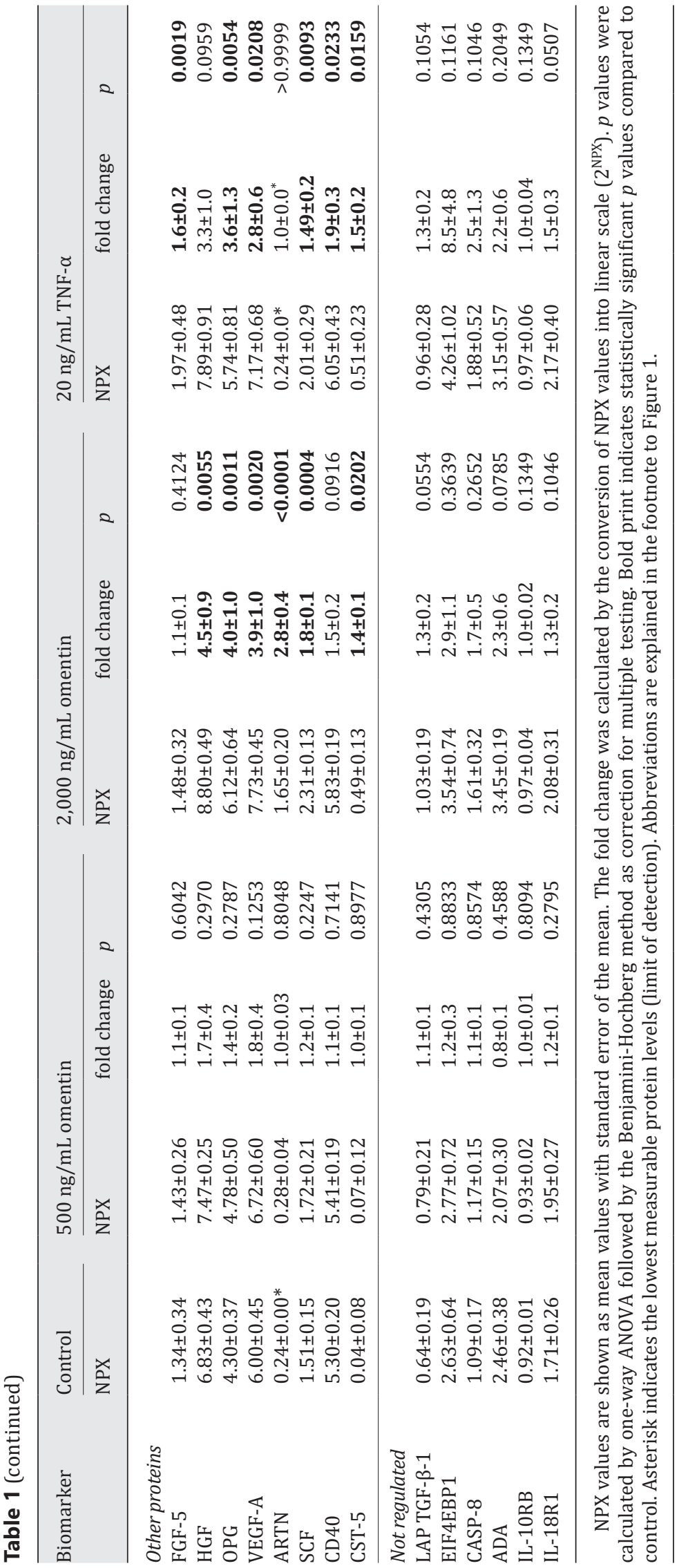


Niersmann et al.: Proinflammatory Action of Omentin in Human Adipocytes

Table 2. Potential upstream activators (top 15) and potential upstream inhibitors of the omentin-induced secretion profile

\begin{tabular}{|c|c|c|c|}
\hline & $\begin{array}{l}\text { Activation } \\
\text { z-score }\end{array}$ & $p$ & Omentin-regulated proteins \\
\hline \multicolumn{4}{|l|}{ Upstream activator } \\
\hline \multicolumn{4}{|l|}{ Cytokines } \\
\hline TNF & +4.13 & $2.32 \times 10^{-22}$ & $\begin{array}{l}\text { CCL11, MCP1, CCL20, MIP1 } \alpha \text {, CCL4, CSF1, CX3CL1, CXCL1, CXCL10, } \\
\text { CXCL11, CXCL5, IL8, IL1 } \alpha, \text { IL6, MMP1, uPA, OPG, VEGF-A }\end{array}$ \\
\hline IL1B & +3.94 & $6.11 \times 10^{-25}$ & $\begin{array}{l}\text { CCL11, MCP4, MCP1, CCL20, MIP1 } \alpha, \text { CCL4, MCP3, CXCL1, CXCL10, } \\
\text { CXCL11, CXCL5, CXCL6, IL8, IL6, MMP1, VEGF-A }\end{array}$ \\
\hline IL1A & +3.06 & $2.29 \times 10^{-29}$ & $\begin{array}{l}\text { MCP1, CCL20, MCP2, CSF1, CXCL1, CXCL10, CXCL5, IL8, IL1 } \alpha, \text { IL6, SCF, } \\
\text { MMP1, MMP10, UPA, OPG }\end{array}$ \\
\hline IL17A & +2.96 & $9.57 \times 10^{-17}$ & CCL11, MCP1, CCL20, CXCL1, CXCL5, CXCL6, IL8, IL1 $\alpha$, IL6 \\
\hline IL18 & +2.95 & $1.33 \times 10^{-16}$ & MCP1, MIP1 $\alpha$, CCL4, CXCL10, CXCL11, IL8, IL1 $\alpha$, IL6, MMP1 \\
\hline \multicolumn{4}{|l|}{ Groups } \\
\hline ERK1/2 & +2.93 & $2.61 \times 10^{-13}$ & MCP1, MIP1 $\alpha$, CCL4, MCP2, IL8, IL1 $\alpha$, IL6, MMP1, VEGF-A \\
\hline P38 MAPK & +2.91 & $8.19 \times 10^{-13}$ & MCP1, MCP2, CXCL1, CXCL10, CXCL11, IL8, IL6, MMP1, VEGF-A \\
\hline ERK & +2.76 & $1.73 \times 10^{-12}$ & MCP1, CCL4, CXCL1, CXCL10, IL8, IL6, LIF, MMP1 \\
\hline \multicolumn{4}{|l|}{ Enzymes } \\
\hline RNASE2 & +3.08 & $8.15 \times 10^{-23}$ & MCP1, MIP1 $\alpha$, CCL4, MCP3, MCP2, CSF1, CXCL10, CXCL5, IL6, IL7 \\
\hline RNASE1 & +2.97 & $2.74 \times 10^{-21}$ & MCP1, MIP1 $\alpha$, CCL4, MCP3, MCP2, CXCL10, CXCL5, IL6, IL7 \\
\hline \multicolumn{4}{|l|}{ Complexes } \\
\hline NFKB (complex) & +3.67 & $1.08 \times 10^{-19}$ & $\begin{array}{l}\text { MCP1, CCL20, MIP1 } \alpha, \text { CCL4, MCP2, CX3CL1, CXCL1, CXCL10, CXCL11, } \\
\text { CXCL6, IL8, IL6, MMP1, uPA }\end{array}$ \\
\hline Immunoglobulin & +2.81 & $3.24 \times 10^{-16}$ & MCP1, MIP1 $\alpha$, CCL4, MCP3, MCP2, CXCL10, CXCL11, IL6 \\
\hline \multicolumn{4}{|c|}{ Transcription regulators } \\
\hline RELA & +3.01 & $9.81 \times 10^{-20}$ & $\begin{array}{l}\text { MCP1, CCL20, MIP1 } \alpha, \text { CXCL1, CXCL10, CXCL11, CXCL5, IL8, IL1 } \alpha \text {, IL6, } \\
\text { MMP1, uPA, VEGF-A }\end{array}$ \\
\hline JUN & +2.91 & $7.81 \times 10^{-19}$ & $\begin{array}{l}\text { MCP1, CCL20, MCP2, CXCL1, CXCL10, CXCL5, IL8, IL1 } \alpha, \text { IL6, MMP1, } \\
\text { MMP10, VEGF-A }\end{array}$ \\
\hline \multicolumn{4}{|l|}{ Kinases } \\
\hline ERBB2 & +2.80 & $7.38 \times 10^{-9}$ & MCP1, CXCL10, IL8, IL1 $\alpha$, IL6, MMP1, uPA, VEGF-A \\
\hline \multicolumn{4}{|l|}{ Upstream inhibitor } \\
\hline \multicolumn{4}{|l|}{ Cytokines } \\
\hline IL1RN & -2.18 & $7.18 \times 10^{-8}$ & CCL20, CXCL11, IL1 $\alpha$, IL6, MMP1 \\
\hline \multicolumn{4}{|l|}{ Groups } \\
\hline Nr1h & -2.20 & $2.14 \times 10^{-10}$ & MCP1, MIP1 $\alpha$, CXCL1, IL8, IL6 \\
\hline \multicolumn{4}{|l|}{ Enzymes } \\
\hline HMOX1 & -2.76 & $9.85 \times 10^{-16}$ & MCP1, CXCL1, CXCL10, CXCL5, IL8, HGF, IL6, VEGF-A \\
\hline
\end{tabular}

The identification of potential upstream regulators was based on the fold changes in expression and $p$ values of omentinregulated proteins that referred to the treatment with 2,000 ng/mL omentin. $p$ values were calculated by Fisher's exact test. The following types of molecules were included in the analysis: complex, cytokine, enzyme, group, growth factor, kinase, peptidase, phosphatase, transcription regulator, translation regulator, transmembrane receptor and transporter. Upstream regulators are ordered based on the highest activation z-score. ERBB2, erb-b2 receptor tyrosine kinase 2; ERK, extracellular signal-regulated kinase; HMOX1, heme oxygenase 1; IL1A, interleukin-1 alpha; IL1B, interleukin-1 beta; IL1RN, interleukin-1 receptor antagonist; IL17A, interleukin-17 alpha; IL18, interleukin-18; JUN, Jun proto-oncogene, AP-1 transcription factor subunit; NFkB (complex), nuclear factor kappa B; Nr1h, nuclear receptor subfamily 1 group H; p38 MAPK, mitogen-activated protein kinase; RELA, transcription factor p65; RNASE1, ribonuclease A family member 1; RNASE2, ribonuclease A family member 2; TNF, tumor necrosis factor. Abbreviations of omentin-regulated proteins IL-18R1, MCP-2, MCP-4, MIP-1 $\alpha$, MMP-1, MMP-10, SCF, TNF, TWEAK, UPA, and VEGF-A are explained in the footnote to Figure 1. 
Niersmann et al.: Proinflammatory Action of Omentin in Human Adipocytes

Omentin Activated Inflammatory Signaling Pathways in Primary Human Adipocytes

We measured phosphorylation levels of key factors of important intracellular inflammatory signaling pathways which were part of identified potential upstream regulators in cell lysates. After 30 -min incubation with $2,000 \mathrm{ng} / \mathrm{mL}$ omentin $(n=3)$, phosphorylation levels of NFkB p65 (Ser536) were increased $7.0 \pm 0.4$-fold ( $p=0.0825)$ ), of p38 MAPK (Thr180/ Tyr182) $18.7 \pm 3.4$-fold ( $p=0.0286$ ), and of ERK1 (Thr202/Tyr204)/ERK2 (Thr185/Tyr187) $3.2 \pm 0.5$-fold $(p=0.0286)$, respectively (Fig. 2). Incubation with $500 \mathrm{ng} / \mathrm{mL}$ omentin had lower effects (Fig. 2).

After a longer incubation period of $24 \mathrm{~h}$, effects were less pronounced. Treatment with $2,000 \mathrm{ng} / \mathrm{mL}$ omentin increased the phosphorylation levels of NFkB p65 (Ser536) $2.1 \pm$ 0.3 -fold ( $p=0.0429$ ), of p38 MAPK (Thr180/Tyr182) 2.6 \pm 0.4 -fold ( $p=0.0429$ ) and of ERK1 (Thr202/Tyr204)/ERK2 (Thr185/Tyr187) 1.8 \pm 0.2 -fold ( $p=0.0212)$, respectively. Treatment of primary human adipocytes with the lower omentin concentration (at $500 \mathrm{ng} / \mathrm{mL}$ ) enhanced the phosphorylation levels of ERK1/2 $2.0 \pm 0.2$-fold ( $p=0.0044)$. Phosphorylation levels of NFkB p65 and p38 MAPK were also higher than in the control, but these increases were not statistically significant (Fig. 3). TNF- $\alpha$ is a potent inducer of the inflammatory NFkB as well as of the p38 MAPK signaling pathways. As shown in Fig. 3A and B, $20 \mathrm{ng} / \mathrm{mL}$ TNF- $\alpha$ increased the phosphorylation levels of NFKB p65 $5.6 \pm 0.9$-fold $(p=0.0007)$ and of p38 MAPK $4.4 \pm$ 1.0 -fold $(p=0.0018)$.

\section{Discussion}

This study found that omentin (i) enhanced the secretion of 30 proinflammatory proteins and (ii) activated proinflammatory intracellular pathways in primary human adipocytes. Thus, omentin appears to induce key features of obesity-associated inflammation, which have been implicated in insulin resistance, T2D, atherosclerosis and CVD.

The Omentin-Induced Secretion Profile Indicates a Proinflammatory Effect of Omentin on Adipocytes

The present study demonstrated that omentin induced the upregulation of proinflammatory cytokines (e.g., IL-6, IL-7, and IL-1 $\alpha$ ) and chemokines (e.g., IL-8, MCP-2, and MCP-3) in the supernatants of differentiated human adipocytes. In addition, we showed that the secretory profile of both omentin and TNF- $\alpha$ highly overlapped because 25 of the omentinregulated biomarkers were also upregulated upon TNF- $\alpha$ administration. Thus, these data argue for overlapping mechanisms by which both proteins affect the secretion of proinflammatory proteins in primary human adipocytes. Whether the increased release of chemokines triggered by omentin, which is almost exclusively detected in visceral fat depots, may contribute to the predominantly visceral immune cell infiltration in adipose tissue of individuals with obesity needs to be elucidated in further studies.

To our knowledge, this is the first experimental study investigating the effect of omentin on the secretion of biomarkers of inflammation in primary human adipocytes and showing that omentin might have a proinflammatory phenotype. These findings extend our recent liquid-chromatography mass spectrometry-based proteomics analysis demonstrating that most of the omentin-regulated proteins and their potential upstream regulators were proinflammatory cytokines and that the omentin-regulated proteins were overrepresented in signaling pathways which might promote a proinflammatory condition [25].

It is important to note that our experimental approach may have been biased due to two aspects. First, the multiplex assay used here contained far more pro- than anti-inflammatory proteins. However, classical anti-inflammatory proteins including IL-4, IL-10, and IL-13 were 
(A)

(C)
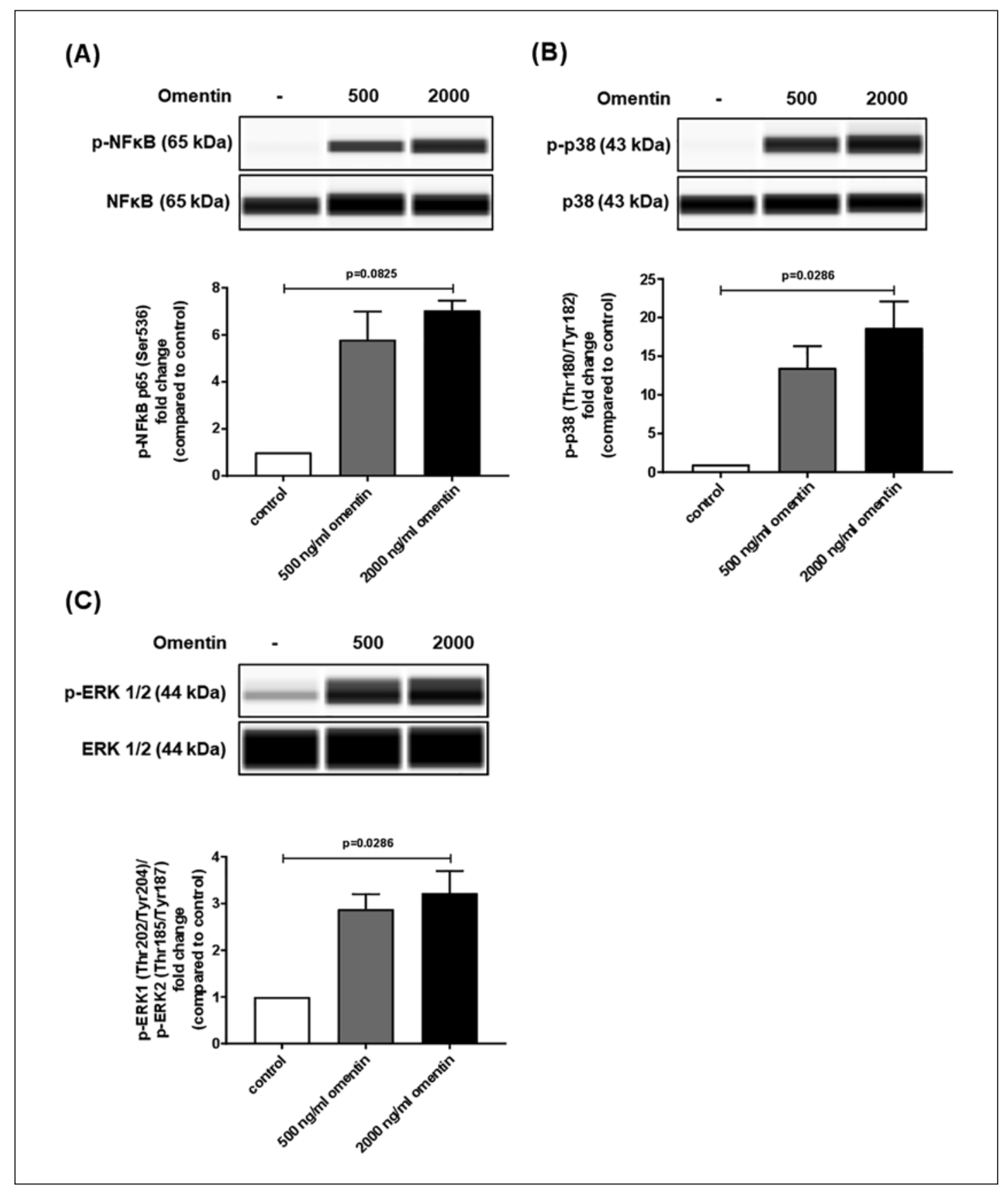

(B)
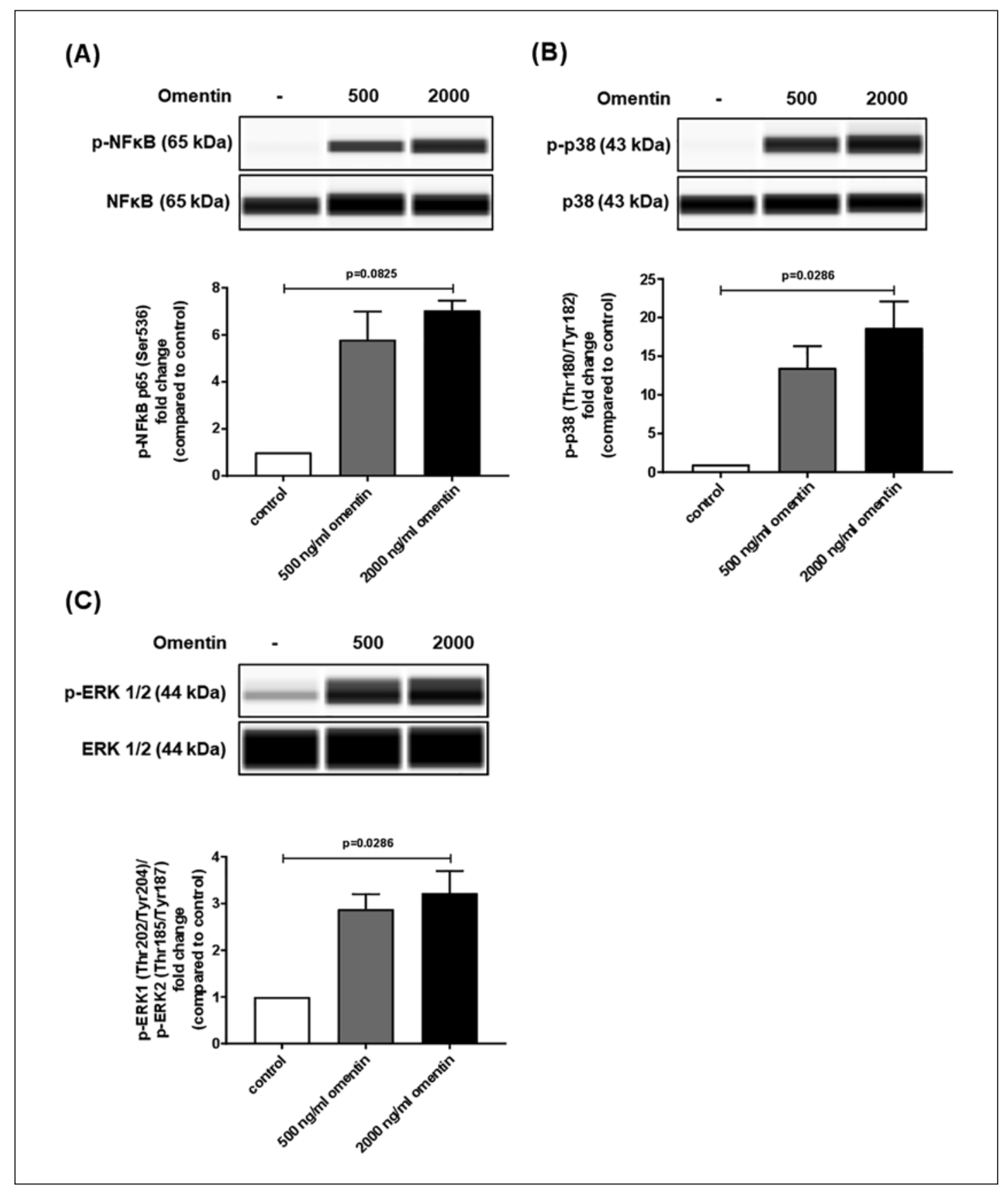

Fig. 2. Impact of short-term omentin treatment ( 500 or 2,000 ng/mL, $30 \mathrm{~min}$ ) on the phosphorylation levels of nuclear factor кB (NFKB) p65 (Ser536) (A), p38 mitogen-activated protein kinase (Thr180/Tyr182) (B), and extracellular signal-regulated kinase (ERK) 1 (Thr202/Tyr204)/ERK 2 (Thr185/Tyr187) (C) in primary human adipocytes. Relative phosphorylation levels of NFKB, p38, and ERK 1/2 were quantified by normalizing to total protein. Expression is shown as fold increase relative to control. Data are shown as the mean \pm SEM $(n=3)$. Differences between treatments were analyzed by Friedman's test followed by Dunn's test as correction for multiple testing. 
(A)
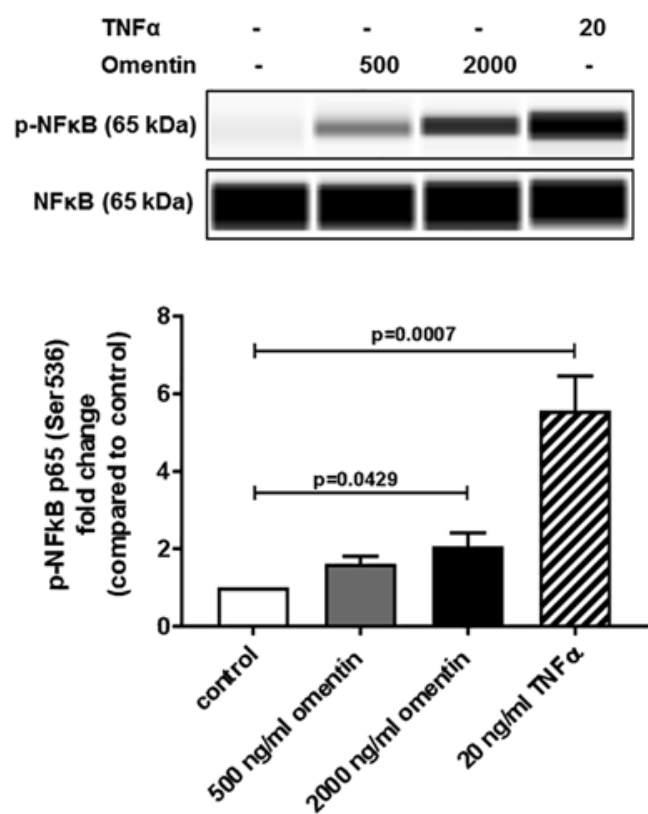

(C)
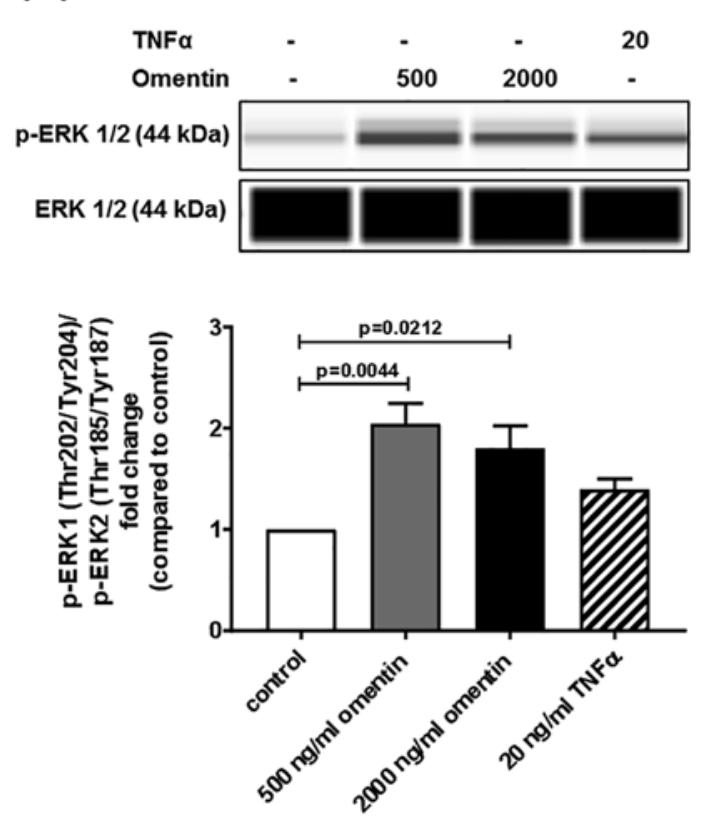

)
(B)
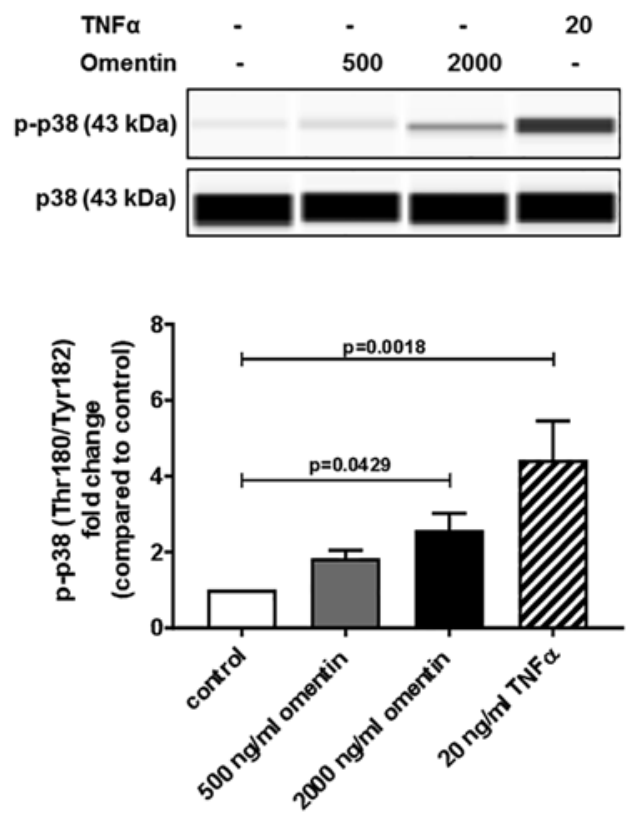
Niersmann et al.: Proinflammatory Action of Omentin in Human Adipocytes

not detectable in supernatants of the control cells and also not upregulated by omentin. Among the omentin-regulated proteins, several proteins such as IL-6, hepatocyte growth factor, and osteoprotegerin also have anti-inflammatory properties depending on the immunological context, so that any discussion with respect to pro- and anti-inflammatory effects represents to some extent an oversimplification if only one cell type is assessed in such in vitro studies.

Second, our approach of using unstimulated adipocytes is less suitable to detect antiinflammatory effects compared to an experimental setting based on lipopolysaccharide (LPS)- or cytokine-activated cells. Therefore, our data may be seen as complementary rather than conflicting compared with other in vitro studies investigating the effect of omentin on inflammatory processes in other cell types.

One study aiming to analyze the relevance of omentin for osteoporosis reported that recombinant omentin (at a supraphysiological concentration of $300 \mu \mathrm{g} / \mathrm{mL}$ ) suppressed the secretion of proinflammatory proteins including IL- $1 \alpha$, IL-1 $1 \beta$, and IL- 6 that was induced by TNF- $\alpha$ in mouse macrophages [31]. The same study evaluated the role of omentin in inflammation using an omentin knockout mouse model and showed that the depletion of omentin led to an increase in serum concentrations of IL-1 $\alpha$, TNF- $\alpha$, and IL-6 [31]. Two additional studies indicated that omentin diminished the levels of proinflammatory cytokines and chemokines including IL-6 and TNF- $\alpha$ in the serum of obese mice [32] or in the lung tissue of LPStreated mice [33]. Thus, these findings argue rather for anti-inflammatory effects of omentin.

These contrasting data might be explained by species- and cell-specific differences in the omentin-induced secretory process because the aforementioned studies only included findings from mice. However, we used primary human adipocytes. In addition, Song et al. administered omentin every 2 weeks for a total of 8 weeks via tail vein injection of an adenoviral vector to obese mice followed by the determination of produced proinflammatory cytokines 2 weeks after the last treatment [32]. In contrast, we measured the levels of proinflammatory biomarkers after the treatment of primary human adipocytes with recombinant omentin for $24 \mathrm{~h}$. Other reasons for the differences might be that Rao et al. [31] used an inflammatory cell model by treating macrophages with TNF- $\alpha$ and that in the study of Qi et al. [33] mice were treated with LPS 3 days after omentin injection. In contrast, we investigated the effect of omentin without a previous or additional proinflammatory stimulus on the secretory process of primary human adipocytes.

Taken together, our results suggest that omentin has mainly proinflammatory properties in our in vitro system of unstimulated primary human adipocytes. Of note, higher levels of several of these biomarkers (e.g., IL-6, IL-8, macrophage inflammatory proteins- $1 \alpha$, MCP-1, vascular end othelial growth factor $\mathrm{A}$, and macrophage colony-stimulating factor 1 ) have been shown to be associated with increased risk of T2D and CVD in large cohort studies and/or meta-analyses [34-38]. These associations may explain the link between higher omentin levels and higher cardiometabolic risk in prospective studies [20-24]. However, it is important to note that our study focused on the impact of omentin on adipocytes and has implications for local effects on adipose tissue, whereas systemic effects of omentin may be more complex.

\section{Omentin May Mediate Its Proinflammatory Action via NFkB, p38 MAPK, and ERK Signaling}

The in silico analysis suggested distinct signaling pathways by which omentin might trigger the release of proinflammatory biomarkers. Several proinflammatory cytokines including TNF- $\alpha$, three members of the IL-1 family (IL1B, IL1A, and IL18), and IL17A were characterized in silico as potential upstream regulators of the omentin-induced secretion profile. TNF- $\alpha$, IL1B, and IL18 are known to induce their downstream signals through activation of several inflammatory kinases including p38 MAPK and ERK as well as the transcription factor NFKB [39-41]. Of note, this transcription factor and the two kinases were 
Niersmann et al.: Proinflammatory Action of Omentin in Human Adipocytes

proposed as potential upstream regulators in our dataset. The fact that the analysis identified the transcription factor RELA (also known as p65) points towards the potential importance of NFKB signaling in the mode of action of omentin. Posttranslational modifications of RELA, which are triggered by the aforementioned cytokines (TNF- $\alpha$ or IL-1) [42], lead to the activation of the canonical NFkB pathway. Our previous proteomics study has already provided preliminary evidence that the activation of NFKB may play a central role in the omentin-regulated secretion pattern [25].

The involvement of these inflammatory signaling pathways in the mode of action of omentin was validated by Western blot analysis. We demonstrated that omentin activated $\mathrm{NFkB}, \mathrm{p} 38$, and ERK pathways in human fat cells. This activation of the signaling pathways is in line with the observed secretion of proinflammatory biomarkers because the transcription factor NFkB reflects a major regulator of immune and inflammatory processes through the regulation of genes encoding proinflammatory cytokines, chemokines, and adhesion molecules [43]. However, it should be noted that NFkB signaling is complex with multiple subunits and proteins involved, so that additional studies would be required to investigate the activation of this pathway in more detail. Another signal transduction pathway that is involved in the regulation of proinflammatory cytokines is the p38 MAPK pathway [44]. Thus, it is conceivable, that these pathways participate in the omentin-induced secretion of proinflammatory biomarkers in primary human adipocytes. In addition, the proinflammatory condition is maintained via a positive feedback loop because the production of inflammatory signals such as IL- $1 \beta$ and TNF- $\alpha$ by the activated pathways serve as stimuli for the same pathways.

In contrast, currently available data from other mechanistic in vitro studies addressed the role of omentin mainly in HUVECs and SMCs. They identified omentin as anti-inflammatory, anti-atherosclerotic and cardioprotective adipokine. In cultured HUVECs, omentin exerted its inhibitory role on the inflammatory state by inhibiting TNF- $\alpha$-induced cyclooxygenase-2 expression via suppressing JNK activation through adenosine monophosphate-activated protein kinase (AMPK)/endothelial nitric oxide synthase (eNOS)-dependent mechanisms [15] and inhibited the expression of intercellular and vascular cell adhesion molecule-1 (ICAM-1 and VCAM-1) through suppression of the ERK/NFKB signaling pathways [16]. Another in vitro study in HUVECs found that omentin enhanced endothelial cell differentiation and reduced apoptotic activity through activation of AMPK/eNOS signaling pathways [13], which in turn argued for protective effects of omentin. In line with these findings, a fourth study in HUVECs reported that omentin promoted vasodilation in isolated blood vessels through endothelium-derived nitric oxide [14]. With respect to vascular SMCs, it has been shown that omentin inhibited the TNF- $\alpha$-induced expression of VCAM-1 and monocyte cell adhesion to SMCs by suppressing p38/JNK signaling pathways [12].

Again, one possible explanation for the discrepancy could be that the regulation of these signaling pathways is dependent on the cell type and tissue. In addition, it is noteworthy that most of the studies have determined the anti-inflammatory effect of omentin in cells pretreated with supraphysiological concentrations of proinflammatory cytokines. In contrast, we investigated whether omentin alone in a physiological concentration has an effect on the inflammatory signaling pathways.

Studies on the role of omentin in inflammation and its mechanism in primary human adipocytes are limited. There is only one study showing that omentin reduced the gene expression of TNF- $\alpha$ in mature adipocytes. However, the study did not report an effect of omentin on the expression of other inflammation-involved genes such as IL- 6 and IL- $1 \alpha$, and other biomarkers such as chemokines were not analyzed [45]. In contrast, the present study examined the impact of omentin on cellular responses at different levels. We could not only show that omentin induced the secretion of proinflammatory biomarkers in adipocyte supernatants, but also that inflammatory signaling pathways were activated upon omentin 
treatment. So far, data from the present study and findings from our previous proteomics analysis [25] addressed the immunomodulatory phenotype of omentin in primary human adipocytes using the most comprehensive approach.

Strengths of the current study include the use of primary human adipocytes. We treated the differentiated adipocytes with recombinant omentin in serum- and growth factor-free medium (in contrast to other studies which used pretreatment with proinflammatory cytokines) in order to determine the specific effect of omentin on basal adipokine release. Although we cannot exclude potential transcriptional effects of FCS used during the differentiation process, it is important to note that all adipocytes were kept under the same conditions, and this should not interfere with the observed effects induced by omentin. Another important strength is the use of physiological concentration of recombinant omentin for stimulation experiments. Finally, we used both in silico and in vitro methods to identify mechanisms that may explain our initial observations with respect to protein expression. Of note, we measured 92 inflammatory biomarkers to comprehensively characterize the omentin-induced alterations in protein abundance in adipocyte supernatants.

Limitations include the use of primary cells from Caucasian donors, and so our results may not be generalizable to other ethnic groups. Moreover, we did not quantify the accumulation of lipids or the differentiation efficacy; so, it is possible that our adipocyte cultures may have contained a low amount of preadipocytes, stem cells, endothelial cells, as well as immune cells despite the in vitro differentiation process. As discussed above, our cell system was better suited to detect pro- rather than anti-inflammatory effects of omentin. In order to assess potential anti-inflammatory effects on adipocytes, different experimental conditions including prestimulated cells may be of interest for future studies.

In conclusion, omentin showed proinflammatory effects on cultured primary human adipocytes through the induction of the secretion of proinflammatory proteins and the activation of the inflammatory pathways NFkB, p38, and ERK1/2. These findings are in line with the positive associations between omentin and risk of T2D and CVD in prospective studies.

\section{Statement of Ethics}

Ethical approval was not required for this study based on the use of commercially available adipocytes.

\section{Disclosure Statement}

The authors declare that they have no conflicts of interest regarding this work.

\section{Funding Sources}

This work was supported by a grant from the German Center for Diabetes Research (DZD; to C. Herder and M. Blüher). The German Diabetes Center is supported by the Ministry of Culture and Science of the state of North Rhine-Westphalia (Düsseldorf, Germany) and the German Federal Ministry of Health (Berlin, Germany). The German Diabetes Center is supported in part by a grant from the German Federal Ministry of Education and Research to the German Center for Diabetes Research (DZD). 
Niersmann et al.: Proinflammatory Action of Omentin in Human Adipocytes

\section{Author Contributions}

C.N., M.B., C.H., and M.C.-K. conceived and designed research; C.N. and K.R. performed experiments; C.N. and M.C.-K. analyzed data; C.N., M.B., M.R., C.H., and M.C.-K. interpreted results of experiments; C.N. prepared figures; C.N. drafted the manuscript; C.N., K.R., M.B., M.R., C.H., and M.C.-K. edited and revised the manuscript; C.N., K.R., M.B., M.R., C.H., and M.C.-K. approved the final version of manuscript.

ORCID

http://orcid.org/0000-0002-2050-093X (C. Herder).

http://orcid.org/0000-0002-3749-3799 (M. Carstensen-Kirberg).

\section{References}

1 Yang RZ, Lee MJ, Hu H, Pray J, Wu HB, Hansen BC, et al. Identification of omentin as a novel depot-specific adipokine in human adipose tissue: possible role in modulating insulin action. Am J Physiol Endocrinol Metab. 2006 Jun;290(6):E1253-61.

2 de Souza Batista CM, Yang RZ, Lee MJ, Glynn NM, Yu DZ, Pray J, et al. Omentin plasma levels and gene expression are decreased in obesity. Diabetes. 2007 Jun;56(6):1655-61.

3 Herder C, Carstensen M, Ouwens DM. Anti-inflammatory cytokines and risk of type 2 diabetes. Diabetes Obes Metab. 2013 Sep;15(s3 Suppl 3):39-50.

4 Herder C, Ouwens DM, Carstensen M, Kowall B, Huth C, Meisinger C, et al. Adiponectin may mediate the association between omentin, circulating lipids and insulin sensitivity: results from the KORA F4 study. Eur J Endocrinol. 2015 Apr;172(4):423-32.

5 Pan HY, Guo L, Li Q. Changes of serum omentin-1 levels in normal subjects and in patients with impaired glucose regulation and with newly diagnosed and untreated type 2 diabetes. Diabetes Res Clin Pract. 2010 Apr;88(1):29-33.

6 Shibata R, Ouchi N, Kikuchi R, Takahashi R, Takeshita K, Kataoka Y, et al. Circulating omentin is associated with coronary artery disease in men. Atherosclerosis. 2011 Dec;219(2):811-4.

7 Wang Q, Feng X, Zhou C, Li P, Kang J. Decreased levels of serum omentin-1 in patients with obstructive sleep apnoea syndrome. Ann Clin Biochem. 2013 May;50(Pt 3):230-5.

8 Zhang Q, Zhu L, Zheng M, Fan C, Li Y, Zhang D, et al. Changes of serum omentin-1 levels in normal subjects, type 2 diabetes and type 2 diabetes with overweight and obesity in Chinese adults. Ann Endocrinol (Paris). 2014 Jul;75(3):171-5.

9 Du Y, Ji Q, Cai L, Huang F, Lai Y, Liu Y, et al. Association between omentin-1 expression in human epicardial adipose tissue and coronary atherosclerosis. Cardiovasc Diabetol. 2016 Jun;15(1):90.

10 Onur I, Oz F, Yildiz S, Oflaz H, Sigirci S, Elitok A, et al. Serum omentin 1 level is associated with coronary artery disease and its severity in postmenopausal women. Angiology. 2014 Nov;65(10):896-900.

$11 \mathrm{Xu}$ T, Zuo P, Cao L, Gao Z, Ke K. Omentin-1 is associated with carotid plaque instability among ischemic stroke patients. J Atheroscler Thromb. 2018 Jun;25(6):505-11.

12 Kazama K, Usui T, Okada M, Hara Y, Yamawaki H. Omentin plays an anti-inflammatory role through inhibition of TNF- $\alpha$-induced superoxide production in vascular smooth muscle cells. Eur J Pharmacol. 2012 Jul;686(1-3): 116-23.

13 Maruyama S, Shibata R, Kikuchi R, Izumiya Y, Rokutanda T, Araki S, et al. Fat-derived factor omentin stimulates endothelial cell function and ischemia-induced revascularization via endothelial nitric oxide synthasedependent mechanism. J Biol Chem. 2012 Jan;287(1):408-17.

14 Yamawaki H, Tsubaki N, Mukohda M, Okada M, Hara Y. Omentin, a novel adipokine, induces vasodilation in rat isolated blood vessels. Biochem Biophys Res Commun. 2010 Mar;393(4):668-72.

15 Yamawaki H, Kuramoto J, Kameshima S, Usui T, Okada M, Hara Y. Omentin, a novel adipocytokine inhibits TNFinduced vascular inflammation in human endothelial cells. Biochem Biophys Res Commun. 2011 May;408(2): 339-43.

16 Zhong X, Li X, Liu F, Tan H, Shang D. Omentin inhibits TNF- $\alpha$-induced expression of adhesion molecules in endothelial cells via ERK/NF-кB pathway. Biochem Biophys Res Commun. 2012 Aug;425(2):401-6.

17 Hiramatsu-Ito M, Shibata R, Ohashi K, Uemura Y, Kanemura N, Kambara T, et al. Omentin attenuates atherosclerotic lesion formation in apolipoprotein E-deficient mice. Cardiovasc Res. 2016 May; 110(1): 107-17.

18 Kataoka Y, Shibata R, Ohashi K, Kambara T, Enomoto T, Uemura Y, et al. Omentin prevents myocardial ischemic injury through AMP-activated protein kinase- and Akt-dependent mechanisms. J Am Coll Cardiol. 2014 Jun; 63(24):2722-33. 
19 Matsuo K, Shibata R, Ohashi K, Kambara T, Uemura Y, Hiramatsu-Ito M, et al. Omentin functions to attenuate cardiac hypertrophic response. J Mol Cell Cardiol. 2015 Feb; 79:195-202.

20 Herder C, Kannenberg JM, Niersmann C, Huth C, Carstensen-Kirberg M, Wittenbecher C, et al. Independent and opposite associations of serum levels of omentin- 1 and adiponectin with increases of glycaemia and incident type 2 diabetes in an older population: KORA F4/FF4 study. Eur J Endocrinol. 2017 Oct;177(4):277-86.

21 Wittenbecher C, Menzel J, Carstensen-Kirberg M, Biemann R, di Giuseppe R, Fritsche A, et al. Omentin-1, adiponectin, and the risk of developing type 2 diabetes. Diabetes Care. 2016 Jun;39(6):e79-80.

22 Menzel J, di Giuseppe R, Biemann R, Wittenbecher C, Aleksandrova K, Pischon T, et al. Omentin-1 and risk of myocardial infarction and stroke: results from the EPIC-Potsdam cohort study. Atherosclerosis. 2016 Aug; 251:415-21.

23 Menzel J, di Giuseppe R, Biemann R, Wittenbecher C, Aleksandrova K, Eichelmann F, et al. Association between chemerin, omentin-1 and risk of heart failure in the population-based EPIC-Potsdam study. Sci Rep. 2017 Oct; $7(1): 14171$.

24 Saely CH, Leiherer A, Muendlein A, Vonbank A, Rein P, Geiger K, et al. High plasma omentin predicts cardiovascular events independently from the presence and extent of angiographically determined atherosclerosis. Atherosclerosis. 2016 Jan;244:38-43.

25 Niersmann C, Hauck SM, Kannenberg JM, Röhrig K, von Toerne C, Roden M, et al. Omentin-regulated proteins combine a pro-inflammatory phenotype with an anti-inflammatory counterregulation in human adipocytes: A proteomics analysis. Diabetes Metab Res Rev. 2019 Jan;35(1):e3074.

26 Herder C, Kannenberg JM, Carstensen-Kirberg M, Strom A, Bönhof GJ, Rathmann W, et al. A systemic inflammatory signature reflecting cross talk between innate and adaptive immunity is associated with incident polyneuropathy: KORA F4/FF4 study. Diabetes. 2018 Nov;67(11):2434-42.

27 Lind L, Ärnlöv J, Lindahl B, Siegbahn A, Sundström J, Ingelsson E. Use of a proximity extension assay proteomics chip to discover new biomarkers for human atherosclerosis. Atherosclerosis. 2015 Sep;242(1):205-10.

28 Nowak C, Sundström J, Gustafsson S, Giedraitis V, Lind L, Ingelsson E, et al. Protein biomarkers for insulin resistance and type 2 diabetes risk in two large community cohorts. Diabetes. 2016 Jan;65(1):276-84.

29 Enroth S, Johansson A, Enroth SB, Gyllensten U. Strong effects of genetic and lifestyle factors on biomarker variation and use of personalized cutoffs. Nat Commun. 2014 Aug;5(1):4684.

30 Carstensen-Kirberg M, Röhrig K, Niersmann C, Ouwens DM, Belgardt BF, Roden M, et al. Sfrp5 increases glucose-stimulated insulin secretion in the rat pancreatic beta cell line INS-1E. PLoS One. 2019 Mar; 14(3):e0213650.

31 Rao SS, Hu Y, Xie PL, Cao J, Wang ZX, Liu JH, et al. Omentin-1 prevents inflammation-induced osteoporosis by downregulating the pro-inflammatory cytokines. Bone Res. 2018 Mar;6(1):9.

32 Song J, Zhang H, Sun Y, Guo R, Zhong D, Xu R, et al. Omentin-1 protects renal function of mice with type 2 diabetic nephropathy via regulating miR-27a-Nrf2/Keap1 axis. Biomed Pharmacother. 2018 Nov;107:440-6.

33 Qi D, Tang X, He J, Wang D, Zhao Y, Deng W, et al. Omentin protects against LPS-induced ARDS through suppressing pulmonary inflammation and promoting endothelial barrier via an Akt/eNOS-dependent mechanism. Cell Death Dis. 2016 Sep;7(9):e2360.

34 Herder C, Baumert J, Thorand B, Koenig W, de Jager W, Meisinger C, et al. Chemokines as risk factors for type 2 diabetes: results from the MONICA/KORA Augsburg study, 1984-2002. Diabetologia. 2006 May;49(5):921-9.

35 Kaptoge S, Seshasai SR, Gao P, Freitag DF, Butterworth AS, Borglykke A, et al. Inflammatory cytokines and risk of coronary heart disease: new prospective study and updated meta-analysis. Eur Heart J. 2014 Mar; 35(9): 578-89.

36 Liu C, Feng X, Li Q, Wang Y, Li Q, Hua M. Adiponectin, TNF- $\alpha$ and inflammatory cytokines and risk of type 2 diabetes: A systematic review and meta-analysis. Cytokine. 2016 0ct;86:100-9.

37 Shore AC, Colhoun HM, Natali A, Palombo C, Khan F, Östling G, et al.; SUMMIT Consortium. Use of vascular assessments and novel biomarkers to predict cardiovascular events in type 2 diabetes: the SUMMIT VIP Study. Diabetes Care. 2018 Oct;41(10):2212-9.

38 Sjaarda J, Gerstein H, Chong M, Yusuf S, Meyre D, Anand SS, et al. Blood CSF1 and CXCL12 as causal mediators of coronary artery disease. J Am Coll Cardiol. 2018 Jul;72(3):300-10.

39 Hata K, Andoh A, Shimada M, Fujino S, Bamba S, Araki Y, et al. IL-17 stimulates inflammatory responses via NF-kappaB and MAP kinase pathways in human colonic myofibroblasts. Am J Physiol Gastrointest Liver Physiol. 2002 Jun;282(6):G1035-44.

40 Sabio G, Davis RJ. TNF and MAP kinase signalling pathways. Semin Immunol. 2014 Jun;26(3):237-45.

41 Stienstra R, Tack CJ, Kanneganti TD, Joosten LA, Netea MG. The inflammasome puts obesity in the danger zone. Cell Metab. 2012 Jan;15(1):10-8.

42 Huang B, Yang XD, Lamb A, Chen LF. Posttranslational modifications of NF-kappaB: another layer of regulation for NF-kappaB signaling pathway. Cell Signal. 2010 Sep;22(9):1282-90.

43 Hoesel B, Schmid JA. The complexity of NF- $\kappa B$ signaling in inflammation and cancer. Mol Cancer. 2013 Aug; 12(1):86.

44 Bachstetter AD, Van Eldik LJ. The p38 MAP kinase family as regulators of proinflammatory cytokine production in degenerative diseases of the CNS. Aging Dis. 2010 Dec;1(3):199-211.

45 Fernández-Trasancos Á, Agra RM, García-Acuña JM, Fernández ÁL, González-Juanatey JR, Eiras S. Omentin treatment of epicardial fat improves its anti-inflammatory activity and paracrine benefit on smooth muscle cells. Obesity (Silver Spring). 2017 Jun;25(6):1042-9. 